\title{
IN SITU DETECTION OF ESR PROTEINS SECRETION DURING MAIZE MICROSPORE EMBRYOGENESIS AND THE SECRETION BLOCKAGE SHOW EFFECTS ON THE CULTURE PROGRESSION
}

\author{
Pilar S. Testillano ${ }^{1}$, María-José Coronado ${ }^{1}$, Anne-Marie Thierry ${ }^{2}$, \\ Elisabeth Matthys-Rochon ${ }^{2}$, María C. Risueño ${ }^{1}$
}

\footnotetext{
${ }^{1}$ Plant Development and Nuclear Architecture. Biological Research Centre, CIB-CSIC, Ramiro de Maeztu 9, E-28040 Madrid, Spain.

${ }^{2}$ Reproduction et Développement des Plantes, ENS Lyon, UMR5667, CNRS/INRA/ ENS/LYON 1, 46 Alleé d'Italie, F-69364 Lyon Cedex 07, France.
}

\section{Running title:}

Esr secretion in maize microspore cultures

Key words: maize, microspore embryogenesis, Esr, signalling, secretion, plant peptides, embryo development, endosperm surrounding region. 


\section{Abstract}

In vitro plant cells in culture release proteins and carbohydrates, but the active molecules responsible for sustaining the switch in embryogenic development and progression have not yet been identified. In maize, the Esr genes encode for small hydrophilic proteins and are expressed in the restricted region of maize endosperm surrounding the embryo, the embryo surrounding region (ESR). In the present work, the possible influence of secreted molecules in the liquid medium during microsporederived embryo development, and specifically the presence of Esr proteins has been analysed in maize microspore cultures. The study has been carried out by in situ monitoring of the structural and cellular organization of developing embryos and the subcellular localization of the Esr proteins by immunofluorescence and immunogold labelling. The results obtained using confocal and electron microscopy revealed that Esr proteins were localized in elements of the secretory pathway and cell walls in microspore-derived embryo cells during early embryogenesis. Esr proteins were also detected in the liquid medium of maize microspore cultures and accumulated at 20 days of culture. Tunicamycin treatment to block protein glycosilation and therefore secretion inhibited microspore-derived embryo development, which was subsequently recovered by supplementation with medium containing all the secreted factors from a welldeveloped microspore culture. Esr labelling was not present in non-developing microspore embryos of cultures treated with tunicamycin, whereas labelling was present again in the Golgi elements and secretory vesicles of embryo cells when development was restored. The results indicate that Esr proteins are part of the secreted proteins, which show a nursing or signalling role during in vitro embryo development in maize 
microspore embryogenesis cultures, and provide new evidence for an endosperm-like function of microspore-derived embryo structures during the early stages. 


\section{Introduction}

Microspore embryogenesis constitutes an intriguing system in which a cell is reprogrammed from its genetically controlled gametophytic programme towards an embryogenic pathway. Isolated microspores or anthers can be cultured in vitro and induced towards embryogenesis by several stress treatments, giving rise to an embryo and a plant. Microspore embryogenesis through in vitro culture is a widely used method to generate genetic variability by obtaining microspore-derived embryos and doublehaploid plants with many applications for plant breeding (Chupeau et al. 1998, Maluszynski et al. 2003). Since microspores are separated from the anther tissues, isolated microspore cultures constitute very convenient systems for studying the mechanisms underlying cell reprogramming and embryo formation (Vicente et al. 1991, Goralsky et al. 2002, Forster et al. 2007), especially since our knowledge of these processes is still largely incomplete. Efficient microspore embryogenic culture systems have been developed in various model species (Touraev et al. 1997, Custers et al. 1994), including cereals like barley (Maraschin et al. 2005, González-Melendi et al. 2005) and maize (Gaillard et al. 1991, Goralsky et al. 2002).More and more information has been reported in recent years of genes and molecules controlling early embryogenic events (Raghavan 2000), but knowledge is still scarce on the genetic control of the process and the possible involvement of external factors regulating embryo growth and development. Difficult access to the very young embryo and endosperm inside the maternal tissues makes analysis of their relationship problematic, though this has been partially overcome with the use of in vitro systems such as somatic and microspore embryogenesis. In carrot somatic embryogenesis initiation of the process depends on the presence of several proteins being secreted into the medium (Van Hengel et al. 
1998), some of which were endochitinases (Van Hengel et al. 1998), as well as in other species like barley (Kragh et al. 1991). The presence of secreted arabinogalactan proteins (AGPs) in maize microspore and zygote cultures has been reported to be a stimulating factor for embryo development (Paire et al. 2003, Borderies et al. 2004). Nevertheless, factors responsible for embryogenesis in established cultures are unknown, and the effects of identified carbohydrates and proteins on in vitro development in plant cultures are not yet understood.

Esr genes, which have been identified in maize (Opshal-Ferstad et al. 1997, Bonello et al 2000, 2002), code for small hydrophilic proteins and their expression has been found specifically in young endosperm, exclusively in the embryo surrounding region (ESR) where highly specialized secretory cells are localized. Different biochemical, molecular and immunolocalization approaches have provided evidence indicating that Esr proteins are secreted by the endosperm cells of the ESR, suggesting a possible nursing role in the exchanges between maternal tissues, endosperm and embryo (Bonello et al. 2002). Another family of genes coding for small hydrophilic proteins, ZmAE1-3, was identified in maize and showed a specific expression in both the ESR and the microspore-derived structures of pollen embryogenesis cultures (Magnard et al. 2000, Testillano et al. 2002, Sevilla Lecoq et al. 2003). More recently, Esr gene expression has been found in developing microspore embryogenic cultures of maize by fluorescence in situ hybridization (FISH), immunofluorescence (IF) and RT-PCR (Massoneau et al. 2005).

In a previous work, an experimental system of inhibition and restoration of maize microspore embryo development using tunicamycin was established (Borderies et al. 2004). Tunicamycin was used as a well-known protein glycosilation inhibitor to 
block secretion and its effect was analysed. Microspore cultures treated with tunicamycin did not develop embryos, whereas the inhibition was cancelled and development restored when the culture medium was removed and replaced with conditioned medium containing all the secreted factors of a well-developed culture of 18-21 days (Borderies et al. 2004).

In this work, Esr protein secretion into the liquid medium of maize microspore cultures and its possible influence on microspore-derived embryo development has been studied. Detection of Esr proteins in the culture medium by immunoblot, monitoring of the structural and cellular organization of developing embryos and in situ subcellular localization of the Esr proteins by immunofluorescence and immunogold labelling were performed at different time periods. This was done in both normal microspore cultures and cultures treated with tunicamycin. The results indicated that the Esr proteins were secreted into the maize microspore cultures and the inhibition of protein secretion affected the development of the microspore-derived embryos. 


\section{Material and Methods}

\section{Plant material}

Maize (Zea mays L.) plant genotypes HD5_HD7and A6, kindly provided by M. Beckert (INRA station,Clermont Ferrand-Theix France), were used to collect tassels for microspore isolation. The plants were raised in a growth chamber under a photoperiod of $16 / 8 \mathrm{~h}$ day/night, at a light intensity of $560 \mathrm{mEm} \_2 \mathrm{~s}-1$ (provided by $400-\mathrm{W}$ high pressure sodium lamps MAZD-MAC $400 \mathrm{E} 40$ ), a day/night temperature of $24 / 19{ }^{\circ} \mathrm{C}$ and humidity of $75 \%$ and $95 \%$, respectively.

\section{Microspore culture}

Microspore isolation and culture techniques were based on the protocol described by Gaillard et al. (1991) but with several changes. In particular, maltose was used instead of sucrose as the carbon source (Goralski et al., 1999, 2002 ).

\section{Collection of conditioned medium from maize microspore cultures}

Collection of conditioned medium was performed as previously described by Borderies et al (2004). Samples of conditioned medium (CM) were collected from normally developing microspore cultures at 18 days.

\section{Tunicamycin inhibition treatment and complementation with conditioned medium} Tunicamycin $(2 \mu \mathrm{g} / \mathrm{ml}$ in $10 \mathrm{mM}$ sodium phosphate in water, $\mathrm{pH} 10.5)$, known to prevent formation of $\mathrm{N}$-linked sugar chains, was used to inhibit protein secretion in the microspore cultures, essentially as described previously (Borderies et al 2004). For the inhibition assays, tunicamycin was added to the microspore culture medium from the 
starting point (time 0) and kept for 18-30 days. For the complementation experiments, in some treated cultures the medium was removed at 18 days and replaced by conditioned medium $(\mathrm{CM})$ from normally developing microspore cultures at the same stage of development. The presence of developing embryos was evaluated for each experiment after $25 / 28$ days (7-9 days after the change of medium).

\section{Immunodetection of Esr in conditioned culture medium}

Anti-Esr polyclonal antibodies (Bonello et al. 2002) raised against purified Esr2 protein fraction and recognizing the three Esr genes identified in maize (Bonello et al. 2000, 2002) were used for immunoblot and immunolocalization assays. The antibody was first tested on immunoblots obtained after 1D SDS-PAGE electrophoresis of proteins extracted from conditioned culture medium at different time points: 5, 14, 20, 28 and 30 days, following the procedure previously described (Borderies et al. 2004). Samples of culture medium were submitted to acetone precipitation and the resulting pellets were re-suspended in SDS-PAGE sample buffer and submitted to denaturation at $100^{\circ} \mathrm{C}$ for 5 min. Total secreted proteins were then separated by SDS-PAGE on $10 \%$ acrylamide slab minigels and electro-transferred onto nitrocellulose sheets. Equal protein content was loaded per well, based on a Lowry protein assay. Anti-Esr antibodies (primary antibodies) were applied to the blots at a dilution of 1: 100 and anti-rabbit IgG-alkaline phosphatase conjugates (Promega, USA) (secondary antibodies) at a dilution of 1: 2500 . Bound antibodies were detected by NBT-BCIP reaction (Nitroblue tetrazolium 5bromo- 4-chloro-3-indolyl phosphate).

Cryoprocessing of samples for immunofluorescence: cryofixation and cryoultramicrotomy 
Samples were cryofixed and cryosectioned for immunofluorescence purposes as previously described (Testillano et al., 2002) so as to better preserve the cell structure and antigenic reactivity. Samples were cryoprotected with $2.3 \mathrm{M}$ sucrose and cryofixed by plunging into liquid propane at $-160^{\circ} \mathrm{C}$ and then stored in liquid nitrogen until cryoultramicrotomy. They were subsequently cut at $-80^{\circ} \mathrm{C}$ to obtain $1 \mu \mathrm{m}$ cryosections which were placed in sucrose drops on aminopropyl-triethoxysilane (Sigma, St. Louis, $\mathrm{MO} / \mathrm{USA}$ ) coated slides and stored at $-20^{\circ} \mathrm{C}$ until used for immunofluorescence assays.

\section{Immunofluorescence}

Frozen slides carrying semi-thin cryosections were thawed at room temperature. Onemicrometer cryosections were washed with PBS to remove the sucrose. Then samples were treated for immunofluorescence as previously described (Testillano et al., 2002, Coronado et al. 2007). ESR antibodies used for the immunofluorescence analysis was immunaffinity purified using the procedure described in Marin-Olivier et al., 2000. They were incubated in $10 \%$ fetal calf serum (FCS) for $10 \mathrm{~min}$, and then diluted $(1 / 10)$ anti-ESR antibody was applied for $1 \mathrm{~h}$ at room temperature. After washing in PBS three times (5 min each), the signal was revealed with green-fluorescent ALEXA fluor 488 conjugated anti-rabbit antibodies (Molecular Probes, Eugene, Oregon, USA), diluted 1:25 in PBS for $45 \mathrm{~min}$ in the dark. After washing in PBS, the sections were counterstained with DAPI, mounted in Mowiol and observed. Confocal optical section stacks were collected using a confocal laser scanning microscope (Leica TCS-SP2). Controls were performed by replacing the first antibody by PBS.

\section{Sample cryoprocessing for immunogold labelling: Lowicryl Embedding}


Samples were fixed in 4\% formaldehyde in phosphate buffered saline (PBS), pH 7.3 at $4^{\circ} \mathrm{C}$ overnight. After washing in PBS, they were dehydrated through a methanol series by progressive lowering of temperature (PLT). Finally all the samples were infiltrated and embedded in Lowicryl $\mathrm{K} 4 \mathrm{M}$ resin at $-30^{\circ} \mathrm{C}$ and polymerized under $\mathrm{UV}$ irradiation. Semithin sections were used to analyse the cellular organization of the embryos under light microscopy. Lowicryl ultrathin sections were mounted on Formvar-coated nickel grids and used for immunogold labelling

\section{Immunogold labelling}

Ultrathin sections were used for immunogold labelling as previously described (Testillano et al., 2002, Coronado et al. 2007). Ultrathin sections from cryoprocessed samples were floated on drops of distilled water, PBS and 5\% BSA in PBS for 5 min. each. They were then incubated with anti-Esr antibodies (immunoaffinity purified, as in the immunofluorescence essays) diluted $1 / 20$, for $1 \mathrm{~h}$ at room temperature. After washing with PBS, the sections were incubated with anti-rabbit secondary antibodies conjugated with 10-nm gold particles (Bio-Cell, Cardiff, UK), and diluted 1/25 in PBS for $45 \mathrm{~min}$. The grids were washed in PBS, rinsed in distilled water and air-dried. Finally the grids were counterstained with $5 \%$ uranyl acetate and $1 \%$ lead citrate and observed in a JEOL 1010 microscope at $80 \mathrm{kV}$. 


\section{Results}

\section{Cell monitoring and development of the embryogenic maize microspore cultures}

Microspore culture for embryogenesis induction in maize was carried out as previously described (Galliard et al 1991) and monitored under inverted and stereo microscopes, where it showed a similar behaviour to that reported in previous works (Magnard et al. 2000, Testillano et al. 2002, Borderies et al. 2004, Massoneau et al. 2005). After inductive pre-treatment of the spikes, unicellular microspores at the vacuolated stage (Fig. 1a) were isolated and put in the corresponding liquid culture medium. A small fraction of the microspores responded to the treatment and developed in vitro, forming multicellular structures or proembryos which could be clearly distinguished after 5 to 7 days and were still surrounded by the microspore wall, the exine (Fig. 1b). Cell proliferation continued and by day 12 most of the proembryos had broken their exine (Fig. 1c) and faster development took place. The round proembryos increased in size and acquired an elongated pear-like shape at 18 days (Fig. 1d). At 30 days, further development resulted in the formation of white structures similar to

embryos (Fig. 1e, f, g) which were able to regenerate plantlets (Galliard et a. 1991, Magnard et al. 2000).

Microspores and microspore-derived structures from selected developmental stages were fixed and processed to analyse their cellular organization by light microscopy observation of semithin sections. At time zero, the isolated microspores showed the typical organization of the vacuolated stage with a large cytoplasmic vacuole and an elongated nucleus at the periphery surrounded by a thin layer of 
cytoplasm (Fig. 2a). 5 to 7 day-old proembryos exhibited a characteristic structure of confinement within the exine and division into two domains with different cell types as previously described and characterized (Testillano et al. 2002). The embryo-like domain contained dense, polygonal cells while the endosperm-like domain contained clear, highly vacuolated cells (Fig. 2b). After release from the exine, the proembryos found at 18 days showed a homogeneous organization with numerous small cells displaying cytoplasmic vacuoles and small nuclei (Fig. 2c). At 30 days, the embryo-shaped white structures showed a heterogeneous organization (Fig. 2d) with dense and clear cells resembling the protodermis and parenchyma of young zygotic embryos.

\section{Analysis of the structural changes in tunicamycin-treated microspore cultures: blocking of development and restoration by conditioned medium}

To analyse the effect of molecules secreted into the medium on microspore development and derived structures, tunicamycin treatment was performed to block protein glycosilation, and therefore secretion. In a previous work it was reported that tunicamycin treatment inhibited embryo development in maize microspore cultures, whereas the substitution of the medium containing the drug after 18 days by a conditioned medium extracted from another 18 days-old normal microspore culture, restored culture progression and embryo formation (Borderies et al. 2004).

In the present work, parallel microspore cultures were studied in control and tunicamycin-containing cultures. The cultures in the normal medium progressed as expected, leading to proembryo formation and embryo structures with the cellular organization described in figures 1 and 2. The cultures containing tunicamycin 
responded to induction but they did not progress correctly, development being stopped after 5/7 days. After induction, in tunicamycin-containing cultures, responsive microspores divided and multicellular proembryos were observed at 5 days (Fig. 3a) with a similar organization as that found in non-treated cultures. Nevertheless, over the following days no significant development was observed and the exine continued to contain the proembryos for much longer than usual. Exine-confined proembryos were observed at 18 days (Fig. 3b) in tunicamycin-treated cultures and no embryos were formed during the subsequent 30-40 days (data not shown)as development was stopped at this time point.

Following the same procedure previously described to restore embryo development (Borderies et al. 2004), conditioned medium was extracted from normallydeveloping microspore cultures at 18 days and used to replace the medium containing tunicamycin in 18 day-old treated cultures. Blocked proembryo development was positively affected, and the proembryos started to grow in size, the exine burst and embryo-like structures (Fig. 3c) appeared 7 days after the elimination of tunicamycin and its replacement by conditioned medium. Other smaller structures were also observed at this time indicating that the restoration of embryogenic development had occurred asynchronically in different proembryos. The shape and organization of these embryos were very similar to that of embryos developed in control cultures at 25-30 days.

Immunodetection of Esr proteins in the liquid medium and in the subcellular compartments of the developing proembryos of the control microspore cultures 
The results of the tunicamycin experiments indicated that some factors present in the medium that were eliminated by the treatment and restored with the conditioned medium were essential to promote embryo development. Since tunicamycin specifically blocked protein glycosilation and secretion, the results also indicated that among these factors there were proteins secreted by the cells in the culture. From among the possible factors which were secreted into the medium, we have studied Esr proteins. In maize zygotic embryogenesis, Esr proteins are secreted by endosperm cells surrounding the developing embryo during the early stages, and it has been suggested that they perform a nursing function (Bonello et al. 2000. 2002). Using specific antibodies raised against maize Esr proteins, we have been able to detect whether they are present in the liquid medium of microspore cultures at different developmental stages by immunoblot of protein extracts from the culture medium. Results are shown in figure 4 where a specific band of approximately $14 \mathrm{kD}$, the expected molecular weight of the Esr proteins, was detected in culture medium of 20 day-old cultures. No similar protein bands were observed at the other developmental stages tested, before that time point: 5 and 14 days, and at later stages: 28 and 30 days (Fig. 4).

Since Esr proteins accumulated in the medium at a culture time of 20 days, their secretion could have occurred during previous developmental stages. In situ localization of the Esr protein was then analysed by immunofluorescence (IF) and immunogold (IG) labelling in 5 and 18 day-old proembryo cells (Fig. 5). Confocal analysis of the IF experiments showed a positive reaction of anti-Esr antibodies at both developmental stages, with a pattern of distribution in small bright foci in the cytoplasms of proembryo cells (Fig. 5 b, e). No IF signal was observed in the nuclei, identified by DAPI staining in the same cryosection (Fig. 5a, d). Interestingly, high fluorescence was observed in the 
cell walls of peripheral cells of the 18 day-old proembryos (fig. 5e), suggesting an accumulation of the protein in the surface of the proembryos which could be explained by a very active secretion of the protein at this developmental stage. Controls replacing the first antibody by a buffered solution did not exhibit fluorescence. Immunogold labelling with anti-Esr antibodies showed a similar distribution pattern in both 5 and 18 day-stages with gold particles appearing in elements of the secretion pathway, e,g, vesicles of different sizes, frequently near the Golgi complex (Fig. 5c, f) and cell walls. Nuclei, vacuoles and other cellular subcompartments did not show significant labelling. Controls without the first antibody produced no significant signal (data not shown).

\section{Immunolocalization of Esr proteins in microspore-derived proembryos of tunicamycin-treated and restored cultures}

We analysed the presence of Esr proteins in the cells of proembryos after 18 days of treatment with tunicamycin by immunogold labelling with anti-Esr antibodies. Even though the general structure of these 18 day-old tunicamycin-treated proembryos seemed to be similar to 5 day-old untreated proembryos, their development stopped at day 5, electron microscopy analysis revealed a different ultrastructural organization with cells showing larger vacuolar and vesicular subcompartments (Fig. 6a). Esr immunogold labelling assays were negative in those proembryos from treated cultures and showed no significant gold particles on any subcellular compartment, including the secretion pathway, vesicles and the cell wall (Fig. 6c). 
The presence of Esr proteins was also analysed in the restored cultures in which the medium containing the tunicamycin had been replaced by a medium of a normally developing culture at a similar time point (18 days). After recovering for several days, the embryo-like structures had developed and displayed well-structured cells with thick cell walls (Fig. 6b). In contrast with the tunicamycin-blocked proembryos, the embryos of restored cultures showed positive anti-Esr immunogold labelling with gold particles decorating the Golgi apparatus and vesicles (Fig. 6d), as well as the cell walls (Fig. 6e), with the same distribution pattern on the secretion pathway as that observed in the normally-developing embryos. 


\section{Discussion}

Plant cells in embryogenic cultures release proteins and carbohydrates, but the active molecules responsible for triggering the switch to embryogenesis and then sustaining its development have not yet been identified, though peptides and oligosaccharides have been identified as signalling molecules in different developmental processes (Bisseling 1999, Sheen et al. 1999, Butenko et al 2009). After fertilization, intercellular signalling events are required between interactive partners, specifically embryo and endosperm (Dreselhauss 2006, Dumas and Rogowsky 2008). In maize, Esr genes encode for small hydrophilic proteins and are expressed in the restricted region of maize endosperm surrounding the embryo, known as the embryo surrounding region (ESR), where highly specialized secretory cells are localized (Opshal-Ferstad et al. 1997, Bonello et al. 2000). Evidence indicating that Esr proteins are secreted by the endosperm cells of the ESR, suggesting a possible nursing role in the exchanges between maternal tissues, endosperm and embryo were reported (Bonello et al. 2002). In a previous work, we demonstrated the existence of $E s r$ gene expression in developing microspore embryogenic cultures of maize by fluorescence in situ hybridization (FISH), immunofluorescence (IF) and RT-PCR (Massoneau et al. 2005). In the present work we analyzed the secretion of the Esr protein by cells of the microspore-derived structures developed in the culture and their presence in the liquid medium, as well as their possible effect on embryo development.

As a result of monitoring the structural and cellular organization of developing embryos and the in situ subcellular localization of the Esr proteins by immunofluorescence and immunogold labelling, we have been able to reveal that Esr 
proteins are localized in various cell structures. These Esr proteins are localized in the secretory pathway and cell walls in microspore-derived embryo cells at different time periods in normal microspore cultures during early embryogenesis, specifically, in multicellular proembryos still surrounded by the exine after 5-7 days of culture, and in larger multicellular embryos formed after exine breakdown at 18-20 days of culture. Results also showed that Esr proteins are present in the liquid medium of maize microspore cultures, accumulating at 20 days of culture. Very low or no expression was reported for Esr proteins in microspore-derived structures at 30 days of culture (Massoneau et al. 2005) by IF, the present results of the immunoblot of the liquid medium agreed with these data and indicated that Esr secretion occurred at early stages of microspore culture, till 18-20 days, the protein accumulating in the liquid medium at this time point, and no expression nor secretion were detected at later stages. Therefore, the Esr protein present in the liquid medium would progressively dilute and was no more detected in the medium at later stages. In contrast, Esr labelling was not found in young microspore proembryos of cultures treated with tunicamycin, a secretion inhibitor; when the tunicamycin was removed, the medium replaced by a conditioned medium, and development restored, Esr proteins were localized, as before, in the Golgi elements and secretory vesicles of embryo cells. Tunicamycin treatment inhibits the secretory pathway and thereby the secretion of signalling molecules triggering embryonic development. The arrest of the Esr protein secretion would be the consequence of the lack of glycosylation of other proteins with essential functions in the secretory pathway. 
From this data, a role for the secreted proteins in sustaining microspore embryo development during the early stages can be deduced. The results clearly showed that Esr proteins are part of these secreted proteins and, therefore, could be responsible, at least in part, of the observed stimulating effects on embryo development in vitro, as indicated by their expression and subcellular localization in various elements of the secretory pathway, a characteristic feature of developing embryos. Furthermore, no Esr expression nor localization in secretory structures was found in arrested microsporederived structures of tunicamycin-treated cultures. However, when microspore-derived embryo development was reactivated by conditioned medium, Esr expression was restored and its subcellular localization was observed in embryo cells.

Several reports demonstrate that plant peptides are important messengers in cell signalling and that secretory peptides and small polypeptides use this signalling activity to regulate various aspects of plant growth and development. Some genes encoding these small secreted polypeptides have been identified in Arabidopsis thaliana and maize (Butenko et al. 2009, Sharma et al. 2003, reviewed in Wang and Fiers 2010). Esr proteins have some structural homology to the developmental signalling secreted peptide CLAVATA3 (CLV3) from A. thaliana, a peptide which was localized in the cells of the ESR and in the apoplast. Moreover, a cell wall invertase inhibitor ( $\mathrm{Zm}$ INVINH1), that plays an important role in regulatory events during kernel development has been shown to share specific features with Esr proteins, notably, their ESR localization, similar and temporal spatial expression, and apoplast targeting (Bate et al. 2004). Previous reports suggested that Esr proteins could function as nursing or signalling molecules allowing differentiated developmental fates for embryo and endosperm (Bate et al. 2004, Bonello et al. 2002), or that they had a defence function, 
protecting the embryo during the earliest stages of development (Balandin et al. 2005). Results revealed that Esr was expressed early on in microspore embryogenesis in 5 to 7 day old multicellular proembryo cells still surrounded by the exine, and Esr expression was also detected at later stages, after exine breakdown, in multicellular embryos after 18 days in culture. Previous work had reported that Esr proteins showed very faint or no signal expression at later microspore embryo stages, in 30 day cultures, by immunofluorescence (Massoneau et al. 2005). The immunoblotting of the liquid medium only detected Esr proteins at 20 days of culture which is compatible with the possible accumulation of smaller quantities of proteins secreted from earlier stages that the immunoblot method could not detect. This timing of expression for Esr in microspore cultures was similar to that occurring in planta where analysis showed Esr expression from 5 DAP (days after pollination) to 15 DAP (Bonello et al. 2000). The expression of endosperm-specific genes early in culture is consistent with the fact that certain genes display a similar dual expression in cereal endosperm and embryo (Olsen et al., 1995; Brown et al.,1996), and suggests a dual potentiality, i.e. the embryo- and endosperm-like "nature" of these young microspore-derived structures.

Our results suggest that Esr proteins are part of the secreted proteins that have a nursing or signalling role in embryo development in vitro and provide new evidence for an endosperm-like function (Testillano et al. 2002, 2004; Massoneau et al. 2005) of microspore-derived embryo structures during early stages of microspore embryogenesis. Taken together, the evidence suggests that for microspore-derived embryo development in vitro, not only is an appropriate cold treatment followed by culture in a sugar-rich medium required, but also the establishment of at least some of the interactions that presumably exist in planta between embryo and endosperm. 
For Review Purposes Only/Aux fins d'examen seulement 


\section{Acknowledgements}

Work supported by projects granted by the Spanish Ministry of Science and Innovation (MICINN) BFU2008-00203 and AGL2008-04255. MJC was a recipient of a postdoctoral contract at the CIB funded by the programme "Juan de la Cierva" of the Spanish MICINN. Thanks are due to Dr. T. Gaude and Dr. P. Rogowsky (ENS, Lyon, France) for kindly providing us with the anti-Esr antibody and for fruitful scientific discussions. 


\section{References}

Balandin M, Royo J, Gomez E, Muniz L, Molina A, Hueros G. 2005. A protective role for the embryo surrounding region of the maize endosperm, as evidenced by the characterisation of ZmESR-6, a defensin gene specifically expressed in this region. Plant Mol. Biol. 58: 269-282.

Bate NJ, Niu XP, Wang YW, Reimann KS, Helentjaris TG. 2004. An invertase inhibitor from maize localizes to the embryo surrounding region during early kernel development. Plant Physiol.134: 246-254.

Bisseling, T. 1999. The role of plant peptides in intercellular signalling. Curr. Opi. Plant Biol. 2: 365-368.

Bonello, J.-F., Opsahl-Ferstad, H.-G., Perez, P., Dumas, C.,Rogowsky, P.M., 2000. Esr genes show different levels of expression in the same region of maize endosperm. Gene 246, 219-227.

Bonello, J.-F., Sevilla-Lecoq, S., Berne, A., Risueno, M.C.,Dumas, C., Rogowsky, P., 2002. Esr proteins are secreted by the cells of the embryo surrounding region. J. Exp. Bot.53, $1559-1568$.

Borderies, G., le Be' chec, M., Rossignol, M., Lafitte, C., leDeunff, E., Beckert, M., Dumas, C., Matthys-Rochon, E. 2004. Characterization of proteins secreted during microspore culture: arabinogalactan proteins (AGPs) stimulate embryo development. Eur. J. Cell Biol. 83, 205-212.

Brown, R.C., Lemmon, B., Doan, D., Linnestad, C., Olsen, O.-A. 1996. The reprogrammed embryo: the endosperm as a quick route to understanding embryogenesis. In: T.L. Wang and A. Cuming (Eds.) Embryogenesis, the Generation of a Plant, BIOS Scientific Publishers, Oxford, pp. 159-172. 
Butenko MA, Vie AK, Brembu T, Aalen RB, Bones AM. 2009. Plant peptides in signalling: looking for new partners. Trends Plant Sci. 14: 255-263.

Chupeau Y, Caboche M, Henry Y. 1998. Androgenesis and Haploid Plants. Springer-Verlag. Berlin. Heidelberg.

Coronado MJ, Testillano PS, Wilson C, Vicente O, Heberle-Bors E, Risueño MC. 2007. The in situ molecular identification of the Ntf4-MAP kinase expression sites in maturing and germinating pollen. Biol. Cell. 99, 209-221.

Custers JBM, Cordewener JHG, Nollen Y, Dons HJM, Campagne MMV. 1994. Temperature controls both gametophytic and sporophytic development in microspore cultures of Brassica-napus. Plant Cell Rep.13, 267-271.

Dresselhaus, T. 2006. Cell-cell communication during double fertilization. Curr. Opi. Plant Biol. 9, 41-47

Dumas, C; Rogowsky, P. 2008. Fertilization and early seed formation, Comptes Rendus Biologies 331, 715-725

Forster, BP; Heberle-Bors, E; Kasha, KJ, Touraev A. 2007. The resurgence of haploids in higher plants. Trends Plant Sci. 12, 368-375.

Gaillard, A, Vergne, P, Beckert, M. 1991. Optimization of maize microspore isolation and culture conditions for reliable plant regeneration. Plant Cell Rep. 10, 55-58.

González-Melendi P, Ramírez C, Testillano PS, Kumlehn J, Risueño MC. 2005. 3D confocal and electron microscopy imaging define the dynamics and mechanisms of diploidization at early stages of barley microspore-derived embryogenesis. Planta, 222, 47-57.

Goralski, G., Lafitte, C., Bouazza, L., Matthys-Rochon, E., Przywara, L., 2002. Influence of sugars on isolated microspore development in maize (Zea mays L). Acta Biol. Cracov. 44, 203-212. 
Goralski, G., Matthys-Rochon, E., Vergne, P., Przywara, L.,1999. Androgenetic development: a fascinating embryo formation process. Acta Biol. Cracov. 41, 51-65.

Kragh KM, Jacobsen S, Mikkelsen JD, Nielsen KA, 1991. purification and characterization of 3 chitinases and one beta-1,3-glucanase accumulating in the medium of cell-suspension cultures of barley (hordeum-vulgare 1). Plant Sci. 76, 65-77.

Magnard JL, Le Deunff E, Domenech J, Rogowsky PM, Testillano PS, Rougier M, Risueño MC, Vergne P, Dumas C. 2000. Genes normally expressed in the endosperm are expressed at early stages of microspore embryogenesis in maize. Plant Mol. Biol. 44, $559-574$.

Maluszynski M, Kasha KJ, Forster BP, Szarejko I. 2003. Doubled haploid production in crop plants, a manual. Kluwer Academic Publishers.

Maraschin, S.F., W. de Priester, H.P. Spaink, M. Wang, 2005. Androgenic switch: an example of plant embryogenesis from the male gametophyte perspective. J. Exp. Bot. 56, 17111726.

Marin-Olivier M, Chevalier T, Fobis-Loisy I, Dumas C, Gaude T. 2000. Aquaporin PIP genes are not expressed in the stigma papillae in Brassica oleracea. Plant J. 24, 231-240.

Massonneau A, Coronado MJ, Audran A, Bagniewska-Zadworna A, Mol R, Testillano PS, Goralski G, Dumas C, Risueño MC, Matthys-Rochon E. 2005. Multicellular structures that develop during in vitro maize pollen embryogenesis express both endosperm- and embryo-specific genes : which is which? Eur. J. Cell Biol. 84, 663-675.

Olsen, O.-A., Brown, R.C., Lemmon, B.E. 1995. Pattern and process of wall formation in developing endosperm. BioEssays 17: 803-812. 
Opsahl-Ferstad, H.-G., Le Deunff, E., Dumas, C. Rogowsky, P.M. 1997. ZmESR, a novel endosperm specific gene expressed in a restricted region around the maize embryo. Plant J. 12: 235-246.

Paire, A., Devaux, P., Lafitte, C., Dumas, C., Matthys-Rochon, E., 2003. Proteins produced by barley microsporas and their derived androgenic structures promote in vitro zygotic maize embryo formation. Plant Cell Tiss. Organ Cult. 73, 167-176.

Raghavan, V. 2000. Developmental Biology of Flowering plants, Springer-Verlag, New York. Sevilla-Lecoq S, Deguerry F, Matthys-Rochon E, Perez P, Dumas C, Rogowsky PM. 2003. Analysis of ZmAE3 upstream sequences in maize endosperm and androgenic embryos. Sex. Plant Reprod. 16, 1-8.

Sharma, VK; Ramirez, J; Fletcher, JC. 2003. The Arabidopsis CLV3-like (CLE) genes are expressed in diverse tissues and encode secreted proteins. Plant Mol. Biol. 51, 415-425.

Sheen J, Zhou L, Jang JC. 1999. Sugars as signaling molecules. Curr. Opin. Plant Biol. 2, 410418.

Testillano PS, Ramírez C, Domenech J, Matthys-Rochon E, Risueño MC. 2002. Young microspore-derived maize embryos show two domains with defined features also present in zigotic embryogenesis. Int. J. Dev. Biol. 46, 1035-1047.

Testillano PS, Georgiev S, Mogensen L, Coronado MJ, Dumas C, Risueño MC, MatthysRochon E. 2004. Spontaneous chromosome doubling results from nuclear fusion during in vitro maize induced microspore embryogenesis. Chromosoma, 112, 342-349.

Touraev, A., Vicente, O., and Heberle-Bors,E. 1997. Initiation of microspore embryogenesis by stress. Trends Plant Sci. 2, 297-302.

Van Hengel, A.J., Guzzo, F., Van Kammen, A., de Vries, S.C. 1998. Expression pattern of the carrot EP3 in suspensión cultures and in developing seeds. Plant Physiol. 117, 43-53. 
Vicente O, Moreno RM, Heberle-Bors E. 1991. Pollen cultures as a tool to study plant development. Cell Biol Rev. 25, 295-306

Wang G, Fiers M. 2010. CLE peptide signalling during plant development. Protoplasma 240, $33-43$. 


\section{Figure Legends}

Figure 1: Monitoring of maize microspore embryogenesis: sequential stages of in vitro cultures showing the development of the microspore-derived embryos. a) Vacuolate microspores at the beginning of culture. b) Multicellular proembryo still surrounded by the exine after 5 to 7 days in culture, DAPI staining showing the nuclei. c) Multicellular proembryo at the time of exine breakdown after 12 days of culture, DAPI staining showing the nuclei. d) Elongated pear-like embryos at 18 days of culture. e, $f, g)$. Embryos formed after 30 days in culture, they show typical embryo shapes in different developmental stages indicating a certain asynchrony. Bars: $50 \mu \mathrm{m}$.

Figure 2: Cellular organization of microspore-derived structures and embryos at different time points in control microspore cultures. Semithin Lowicryl sections. a) Vacuolate microspores at the beginning of culture. b) Multicellular proembryo still surrounded by the exine, after 5 to 7 days in culture. c) Elongated embryos after 18 days in culture. d) Embryos after 30 days in culture. Bars: a: $10 \mu \mathrm{m}, \mathrm{b}, \mathrm{c}, \mathrm{d}: 50 \mu \mathrm{m}$.

Figure 3: Cellular changes in microspore cultures treated with tunicamycin. Semithin Lowicryl sections. a) Multicellular proembryo with exine after 5 days in tunicamycin-treated cultures showing a similar organization than that of non-treated 5 day-old cultures. b) Multicellular proembryo after 18 days of treatment with tunicamycin, the exine is still surrounding the proembryo indicating development blockage. c) Embryo development after 7 days of tunicamycin elimination and supplementation with a conditioned medium. Bars: $50 \mu \mathrm{m}$. 
Figure 4: Immunoblot of Esr in protein extracts of the culture medium at different time points. Lines 1-6 correspond to collection days of the liquid medium: 5/7, 14, 20, 28, 30 and 34 days respectively. Arrow points out a band of approximately $14 \mathrm{kD}$, the expected MW of Esr proteins, in medium extracts of 20 days in culture.

Figure 5: Esr immunolocalization in normally developing microspore embryogenesis cultures. $a, b, d, e)$ Immunofluorescence on semithin cryosections of proembryos after 5 days $(a, b)$ and 18 days $(d, e)$ in culture. a, d: DAPI staining showing the nuclei of the same cryosection as in b, e which show the Esr immunofluorescence signal on small cytoplasmic spots. c, f) Immunogold labelling on ultrathin Lowicryl sections, cytoplasmic regions of embryo cells of 5 days (c) and 18 days (f), gold particles show a pattern of localization over the secretory pathway elements, e.g. Golgi (G) elements, vesicles (v) and cell walls (CW). N: nucleus, Ct: cytoplasms. Bars: a, b, c, d: $50 \mu \mathrm{m}$, e, f: $0,2 \mu \mathrm{m}$.

Figure 6: Esr immunogold labelling in microspore-derived structures in tunicamycin-treated cultures and restored cultures. a) Ultrastructure of a proembryo after 18 days of tunicamycin treatment which blocks its development showing the exine (Ex) at the periphery. b) Ultrastructure of a proembryo from a restored tunicamycintreated culture, after 7 days of tunicamycin elimination and supplementation with conditioned medium. c) High magnification of a cytoplasmic region (square $\mathrm{c}$ in figure a) of embryo after 18 days of tunicamycin treatment showing no immunogold labelling, d, e) High magnification of cytoplasmic regions (squares d, e, in figure b) of embryos from restored cultures displaying immunogold labelling in Golgi (G) vesicles and 
elements of the secretory pathway. CW: cell wall, $\mathrm{Ct}$ : cytoplasms, v: vesicle. Bars: $\mathrm{a}, \mathrm{b}$ : $5 \mu \mathrm{m}, \mathrm{c}, \mathrm{d}, \mathrm{e}: 0,2 \mu \mathrm{m}$. 

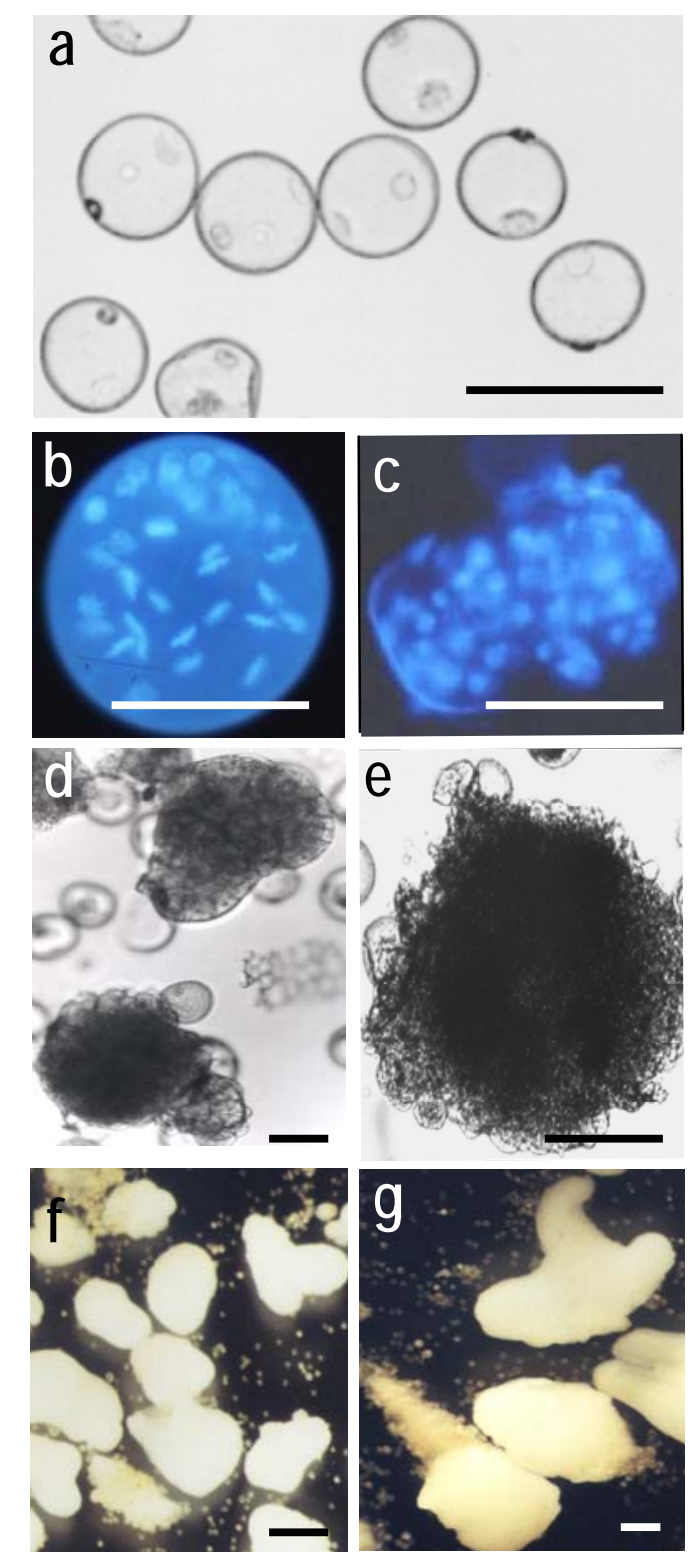

Figure 1 

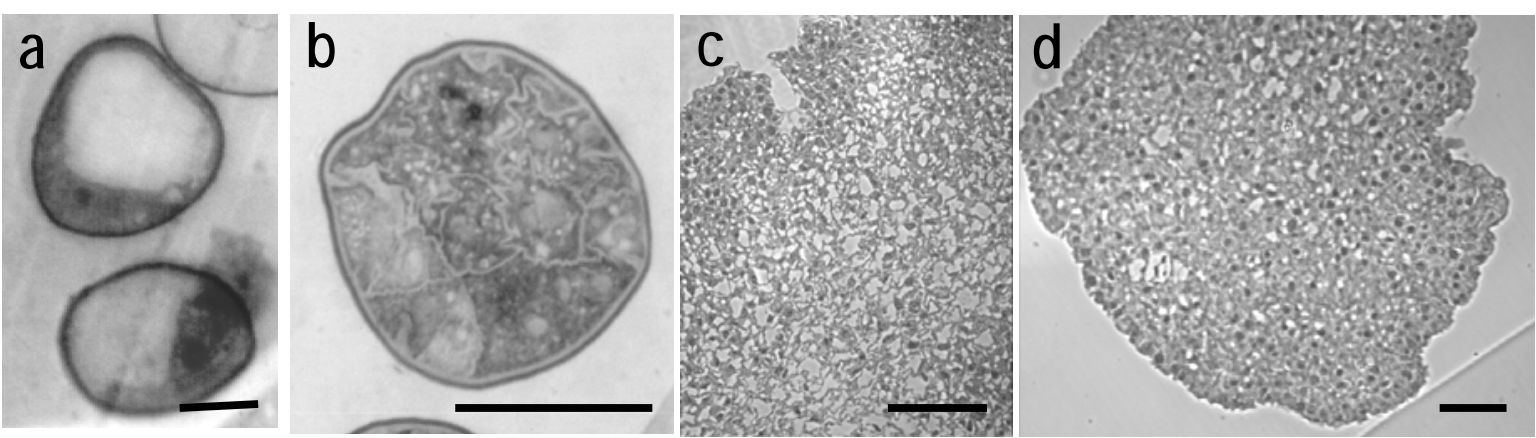

Figure 2 

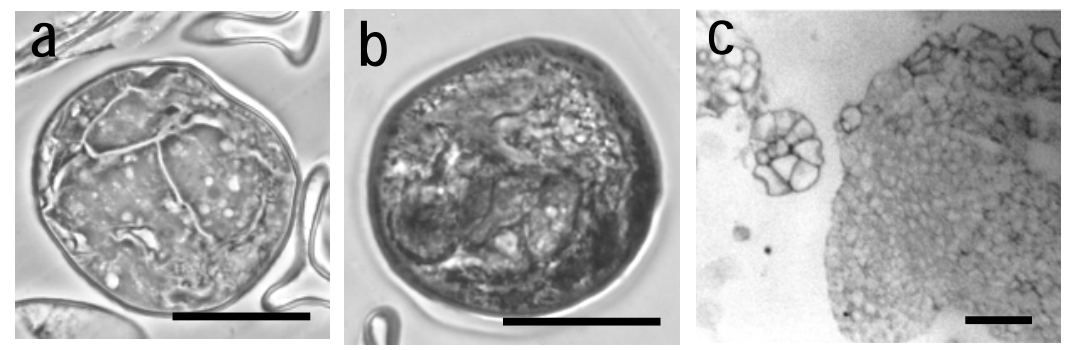

Figure 3 


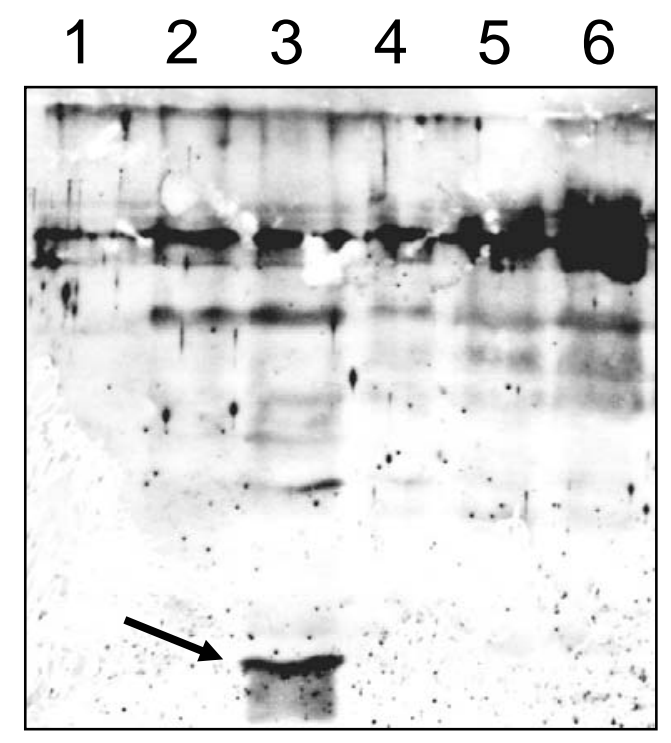

Figure 4 

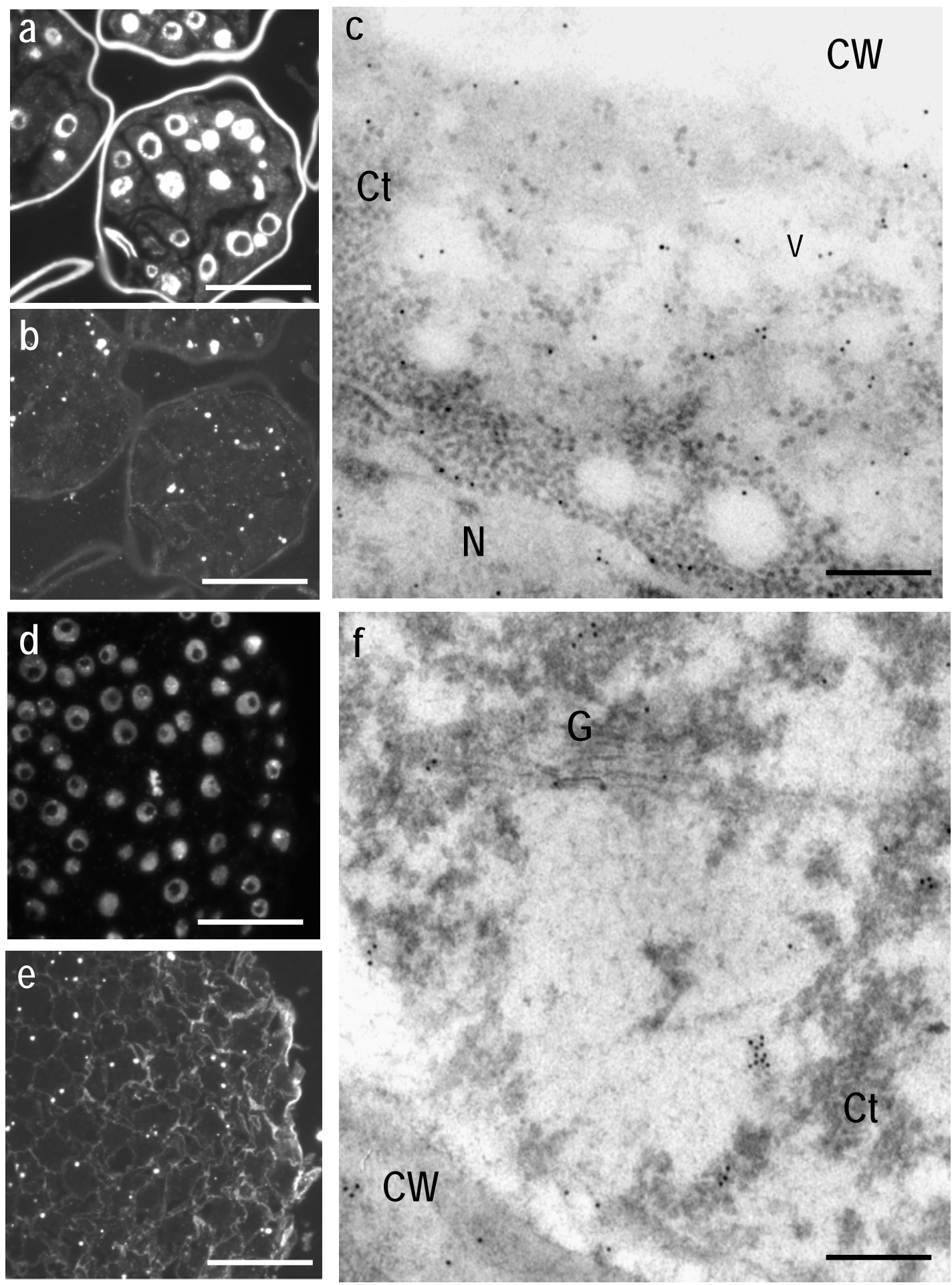

Figure 5 

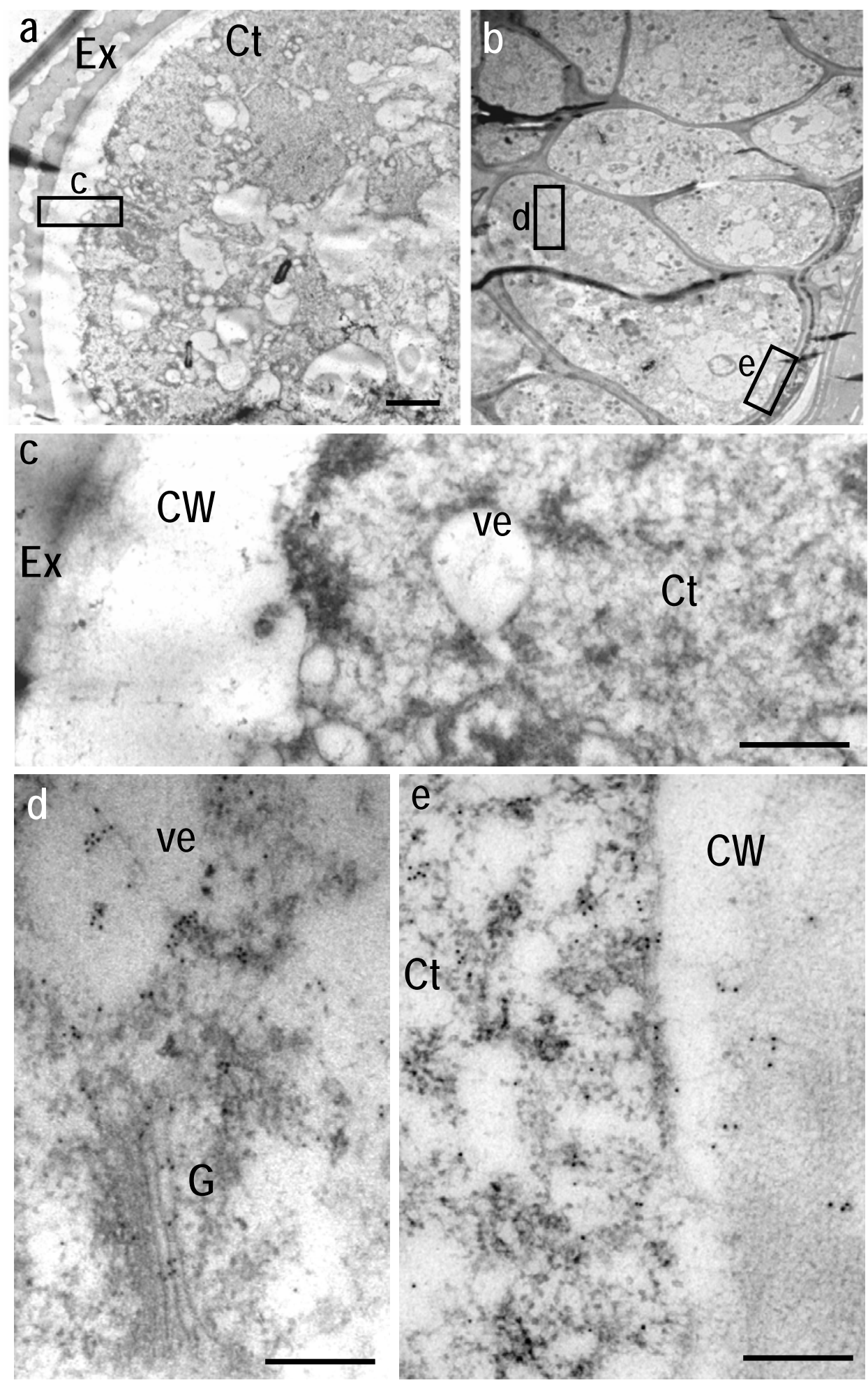

Figure 6 